\title{
Measuring the Optical Absorption of Single Nanowires
}

\author{
Milo Yaro Swinkels $\odot,{ }^{1}$ Alessio Campo, ${ }^{1}$ Daniel Vakulov@ ${ }^{2}$ Wonjong Kim, ${ }^{3}$ Luca Gagliano, ${ }^{2}$ \\ Simon Escobar Steinvall, ${ }^{3}$ Hermann Detz $\odot,{ }^{4,5}$ Marta De Luca $\odot,{ }^{1}$ Alois Lugstein $\odot,{ }^{5}$ Erik Bakkers, ${ }^{2}$ \\ Anna Fontcuberta i Morral ${ }^{3,3}$ and Ilaria Zardo ${ }^{1, *}{ }^{*}$ \\ ${ }^{1}$ Department of Physics, University of Basel, 4056 Basel, Switzerland \\ ${ }^{2}$ Department of Applied Physics, Eindhoven University of Technology, 5600 MB Eindhoven, Netherlands \\ ${ }^{3}$ Institute of Materials, Faculty of Engineering, École Polytechnique Fédérale de Lausanne, Lausanne, Switzerland \\ ${ }^{4}$ Central European Institute of Technology, Brno University of Technology, 61200 Brno, Czech Republic \\ ${ }^{5}$ Institute of Solid-State Electronics and Center for Micro- and Nanostructures, TU Wien, 1040 Vienna, Austria \\ ${ }^{6}$ Institute of Physics, Faculty of Basic Sciences, École Polytechnique Fédérale de Lausanne, Lausanne,
} Switzerland

(Received 14 February 2020; revised 16 June 2020; accepted 31 July 2020; published 17 August 2020)

In this work we present a method to quantitatively measure the optical absorption of single nanowires that can be applied over a wide range of temperatures and with a high enough sensitivity to enable the measurement of below-band-gap absorption (as well as the absorption of single molecules). The method is based on accurately measuring the heat flow coming from a nanowire when it is illuminated by a laser beam. We experimentally verify this method by measuring the absorption of both a zincblende and a wurtzite GaAs, a wurtzite GaP, and a superlattice $\mathrm{Zn}_{3} \mathrm{P}_{2}$ nanowire. Furthermore, we find that the $\mathrm{Zn}_{3} \mathrm{P}_{2}$ nanowires have the largest absorption of all these materials. We analyze the advantages and disadvantages of the method and study its range of applicability.

DOI: 10.1103/PhysRevApplied.14.024045

\section{INTRODUCTION}

In recent years, nanowires (NWs) have emerged as promising building blocks for applications in the field of electronics [1-3], energy conversion [4,5], and optoelectronics [6-8], among others $[9,10]$. The wide range of applicability of NWs stems first of all from their geometry, which can alter mechanical, electronic, as well as optical effects [11,12], but also from the possibility to grow materials with crystal structures that are unstable in bulk [13-15] and have different electronic [16,17] and phononic $[18,19]$ properties from their bulk counterparts.

The possibility to engineer the optical response of NWs has led to enhanced performances in photovoltaics $[12,20]$, light emission [6,16], and photoelectrochemical cells $[9,21]$. Besides these applications, another field in which it is crucial to measure absorption is Raman thermometry, widely used to assess the thermal conductivity of single NWs. Therein, the absorbed power is usually only simulated, which is the main drawback of the technique [22]. In this context, a quantitative assessment of the optical absorption in nanostructures, in particular in NWs, is essential to measure the absorption and experimentally probe effects coming from optical

\footnotetext{
*ilaria.zardo@unibas.ch
}

resonances, band-structure alterations, or experimental conditions that often cannot be properly taken into account by simulations. This is due to the fact that either simulations cannot be carried out (they can be done only in wellknown material systems as they require the knowledge of the energy dependence of the refractive index, which is experimentally unknown, e.g., in many for wurtzite III-V NWs), or they can lead to inexact results, as we discuss in this paper. In this frame, the optical absorption is defined as the fraction of incoming light that is not transmitted, reflected, or re-emitted after interacting with the NW $\left(\alpha=P_{\text {abs }} / P_{\text {in }}\right)$, as is commonly done with this type of measurement [23-27].

Several quantitative methods to measure the optical absorption of single nanostructures (including NWs) [2327] or even single molecules [28,29] have been developed, but since all of these have their limitations and often require complicated measurement schemes, measurements on single NWs often rely on PL excitation (PLE) or photocurrent measurements and are thus qualitative in nature $[8,11]$. In this work, we present a method for measuring the optical absorption of semiconductor NWs with very high sensitivity, and which can be applied in the full temperature range down from $\mathrm{mK}$ up to the maximum stable temperature of the measured material, at variance with other methods to measure absorption, that have to 
be applied at [25] or near room temperature [24,26]. As a proof of principle, we show measurements performed at $70 \mathrm{~K}$ and at room temperature (due to slight heating by the laser the actual temperature at the laser spot is estimated to be at most $70 \mathrm{~K}$ above the measurement temperature at room temperature, and at most $20 \mathrm{~K}$ for the $70-\mathrm{K}$ measurement). By replacing the $\mathrm{Pt}$ in the resistive thermometers with, e.g., $\mathrm{NbN}$, measurements can in principle be done even at a few $\mathrm{mK}$ [30]. A similar approach has previously been used to measure the absorption of carbon nanotube bundles [23], but in this work we simplify the geometry (which enhances sensitivity as well as simplifies fabrication), apply it to semiconductor nanowires, and demonstrate the versatility by extending the wavelength range. Furthermore, we also perform a more detailed analysis showcasing the range of applicability of this type of measurement.

We test the method by measuring the optical absorption of a zincblende (ZB) GaAs NW and compare the results with finite-difference time domain (FDTD) simulations using the MEEP software package [31]. To verify the influence of the crystal structure, we also measure a GaAs NW with wurtzite (WZ) crystal structure. It is worth highlighting that GaAs is a material used in light-emitting diodes (LEDs) [32], and solar cells [20], and thus its optical properties have been extensively studied. Furthermore, we measure the absorption in WZ GaP NWs. This crystal structure has recently been obtained in the NW geometry and is demonstrated to have a pseudodirect band gap [33], unlike the bulk ZB structure that has an indirect band gap. The band gap of this material has been measured in PL measurements at $4 \mathrm{~K}$ to be $2.19 \mathrm{eV}$ [34]: however, the room-temperature band gap still remains to be verified. If we consider typical temperature dependence of semiconductor band gaps, it will most likely fall within our measurement range of $488-633 \mathrm{~nm}$. Therefore, this measurement not only allows learning more about this material but also testing the limits of the method by measuring absorption below the band gap. Finally, we measure the absorption in a $\mathrm{Zn}_{3} \mathrm{P}_{2}$ superlattice NW. Owing to its strong absorption in the visible range and earth abundancy, $\mathrm{Zn}_{3} \mathrm{P}_{2}$ is a promising material for photovoltaics, which has recently been grown epitaxially in the NW form [35]. Our measurements testify its great capability to absorb light. In bulk an indirect band gap has been measured at $1.38 \mathrm{eV}$ and a direct band gap at $1.5 \mathrm{eV}$ [36], while a transition at $1.43 \mathrm{eV}$ has been measured using CL measurements [35], so all our measurements are at an energy far beyond the band gap, but in the interesting range for photovoltaic applications.

This paper is structured as follows. First the details of the method are explained in Sec. II. Then the experimental results are described in Sec. III. Finally a thorough comparison with existing methods is given in Sec. IV.

\section{EXPERIMENTAL DETAILS}

\section{A. Measured nanowires}

The ZB and WZ GaAs NWs are grown using selfcatalyzed vapor-liquid-solid (VLS) growth on Si substrates using MBE. For more information on the growth of the ZB NWs see Ref. [3], while for the growth of the WZ NWs see Ref. [37]. Both types of GaAs NWs have an average diameter of $70 \pm 5 \mathrm{~nm}$, as measured using SEM. The WZ GaP NWs are synthesized using Au-catalyzed VLS growth in an MOVPE reactor. They had a predefined and verified diameter of 50 and $75 \mathrm{~nm}$. For more information see Ref. [16]. The $\mathrm{Zn}_{3} \mathrm{P}_{2}$ NWs are grown using substrate-mediated VLS growth on an $\operatorname{InP}(100)$ substrate using MBE. These wires exhibit a superlattice (SL) structure and an average diameter of $100 \pm 10 \mathrm{~nm}$, with a slight tapering. The growth of these NWs has been described in more detail in Ref. [35].

\section{B. Absorption measurements}

The measurements are performed using a suspended microplatform [38] commonly used for probing thermal transport in NWs [39-42]. The platform consists of two suspended $\mathrm{SiN}_{x}$ membranes supporting a platinum meander. Since the resistance of platinum depends linearly on the temperature, these elements can be used as heater and thermometer. The devices are operated at pressures of the order of $10^{-5}$ mbar, ensuring that both convective and radiative heat losses are at least 3 orders of magnitude below the conductive losses [43] so that all absorbed power is transported to the environment via conduction through the suspended $\mathrm{SiN}_{x}$ beams. For the absorption measurements described in this work, a single NW is suspended from one of the $\operatorname{SiN}_{x}$ membranes without touching the membrane across. This geometry ensures negligible levels of strain on the NW [44]. A laser is focussed on the NW using a microscope objective. Since the device is in steady state during these measurement, the incoming energy has to be equal to the energy flowing out. The incoming energy is given by the absorbed power, while the outgoing energy can, in general, be either thermal (heat conduction), electronic (photocurrent), or radiative (thermal radiation and re-emission) in nature. Since electronic losses can be excluded by the isolating properties of the $\mathrm{SiN}_{x}$, thermal radiation is estimated to be several orders of magnitude lower than conduction, and re-emission is not counted towards the absorbed power in our current definition, the only way the energy can go out of the system is through thermal conduction. The absorbed power causes an increase in temperature of the membranes, which is measured using the platinum resistive thermometer (PRT) (see Fig. 1). Assuming all heat is transported out through the $\mathrm{SiN}_{x}$ nanobeams, the increase in temperature is given 


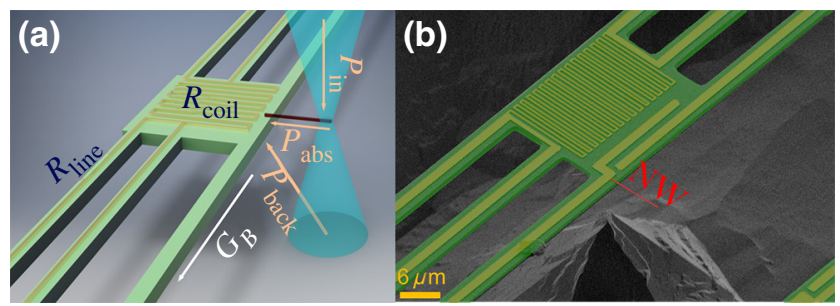

FIG. 1. (a) Schematic of the proposed measurement scheme (not in scale). A laser is focussed on a NW suspended in a cantilevered configuration from a $\mathrm{SiN}_{x}$ platform with a resistive thermometer. Part of the incoming laser power $P_{\text {in }}$ is absorbed $\left(P_{\text {abs }}\right)$ while another part $\left(P_{\text {back }}\right)$ is scattered from the silicon substrate. The absorbed power flows through the NW to the platform causing a temperature increase, and a subsequent increase of the resistance of the thermometer $R_{\text {coil }}$, proportional to the heat conductance to the environment $G_{B}$. This change in resistance can be measured and can be directly related to the absorbed power. (b) False-color SEM image of the device with the $\mathrm{SiN}_{x}$ in green, the Pt contacts in yellow, and the NW in red.

by Fourier's law:

$$
P_{\mathrm{abs}}=G_{b}\left(T_{\text {coil }}-T_{0}\right),
$$

with $P_{\text {abs }}$ the absorbed power, $G_{b}$ the beam conductance, $T_{\text {coil }}$ the coil temperature as measured using the PRT and $T_{0}$ the environment temperature. As seen from the above equation, the measurement itself does not depend on specific NW characteristics such as the thermal conductivity of the NW. This means that the device can be calibrated separately and can then be used for a wide range of NWs. To test this hypothesis, a homogeneous NW is measured on different positions along the NW axis, which alters the thermal conductance through the NW, but does not alter the absorbed power measured. Indeed the same absorption is found on different positions of the NW, using the same calibration (see Appendix A).

The calibration is performed using a higher current through the PRT so that the heating effect cannot be ignored. This gives a relation between the resistance and the dissipated power. However, in this case the current is also flowing through the connecting platinum lines and power is also dissipated in these lines. Assuming a linear temperature profile along the lines (a common assumption for this kind of device $[38,45]$ ), half of the power dissipated in the lines contributes to the heating of the membranes, while the other half is lost to the environment. The calibration needs to be corrected with a constant factor, since in the real experiment all power is absorbed in the membrane:

$$
P_{\text {coil }}=I^{2}\left(R_{\text {coil }}+\frac{1}{2} R_{\text {line }}\right)=P_{\text {total }} \times \frac{R_{\text {coil }}+\frac{1}{2} R_{\text {line }}}{R_{\text {coil }}+R_{\text {line }}}
$$

where $P_{\text {coil }}$ is the power dissipated in the coils, $R_{\text {coil }}$ and $R_{\text {line }}$ are the electrical resistance of the coil and the lines, respectively, (the relevant resistances can be determined separately by using both a four-point and a two-point measurement) and $P_{\text {total }}$ is the total dissipated power during the calibration [given by $I^{2}\left(R_{\text {coil }}+R_{\text {line }}\right)$ ]. Since the resistance has a linear dependence on temperature, Eq. (1) can be rewritten as:

$$
P_{\mathrm{abs}}=\frac{d P_{\text {coil }}}{d R_{\text {coil }}} \times \Delta R
$$

where $d P_{\text {coil }} / d R_{\text {coil }}$ is determined using the device calibration and $\Delta R$ is the change in resistance when turning the laser on. In this way the exact temperature of the coil, nor the wire needs to be known, avoiding the need for an exact determination of the temperature coefficient of resistance and the conductance of the beams Gb from Eq. (1).

After calibration, a NW is placed in a cantilevered configuration from one platform. This is done using a hydraulic micromanipulator under an optical microscope, to prevent any contamination or degradation from SEM of the NW [46]. Then the sample is loaded in a vacuum chamber with optical access and the resistance of the PRTs is measured using a sufficiently low electrical power to ignore heating effects in the PRTs. For this project several different laser wavelengths are used (a 488-nm solid-state sapphire laser, an $\mathrm{Ar}^{+}$ion laser tuned to 514 $\mathrm{nm}$, an $\mathrm{Ar}^{+} / \mathrm{Kr}^{+}$laser tuned to $568 \mathrm{~nm}$, and a HeNe laser at $633 \mathrm{~nm}$ ). The laser is focused on the sample using a numerical aperture $0.5100 \times$ objective giving a diffraction limited spot size. The laser (power $20 \mu \mathrm{W}$ before objective, approximately $12 \mu \mathrm{W}$ after objective, depending on wavelength) is turned on and off periodically and the resistance is averaged over approximately $30 \mathrm{~s}$ to get the resistance in the "laser on" state as well as the "laser off" state. While the laser is off the laser spot is moved by $50 \mathrm{~nm}$ and then the procedure is repeated [see Fig. 2(a)]. In this way the peak power, namely the power absorbed when the laser is hitting the center of the NW, is extracted for different wavelengths and polarizations, as shown in Fig. 2(b) for a representative set of parameters. The absorptance is then calculated as the absorbed power divided by the total incident power. Note that here the incident power is the total power coming into the cryostat (as is done in, e.g., Ref. $[25,47]$ ), not just the part of the power directly hitting the NW. This is done because in our experiments the wavelength is larger than the NW diameter, and thus the influence from diffraction cannot be ignored. This means that photons out of the NW area could be absorbed, while photons within the NW area might not irradiate the NW surface. Using the total incident power also facilitates an easy comparison with other experiments and for engineering applications. 


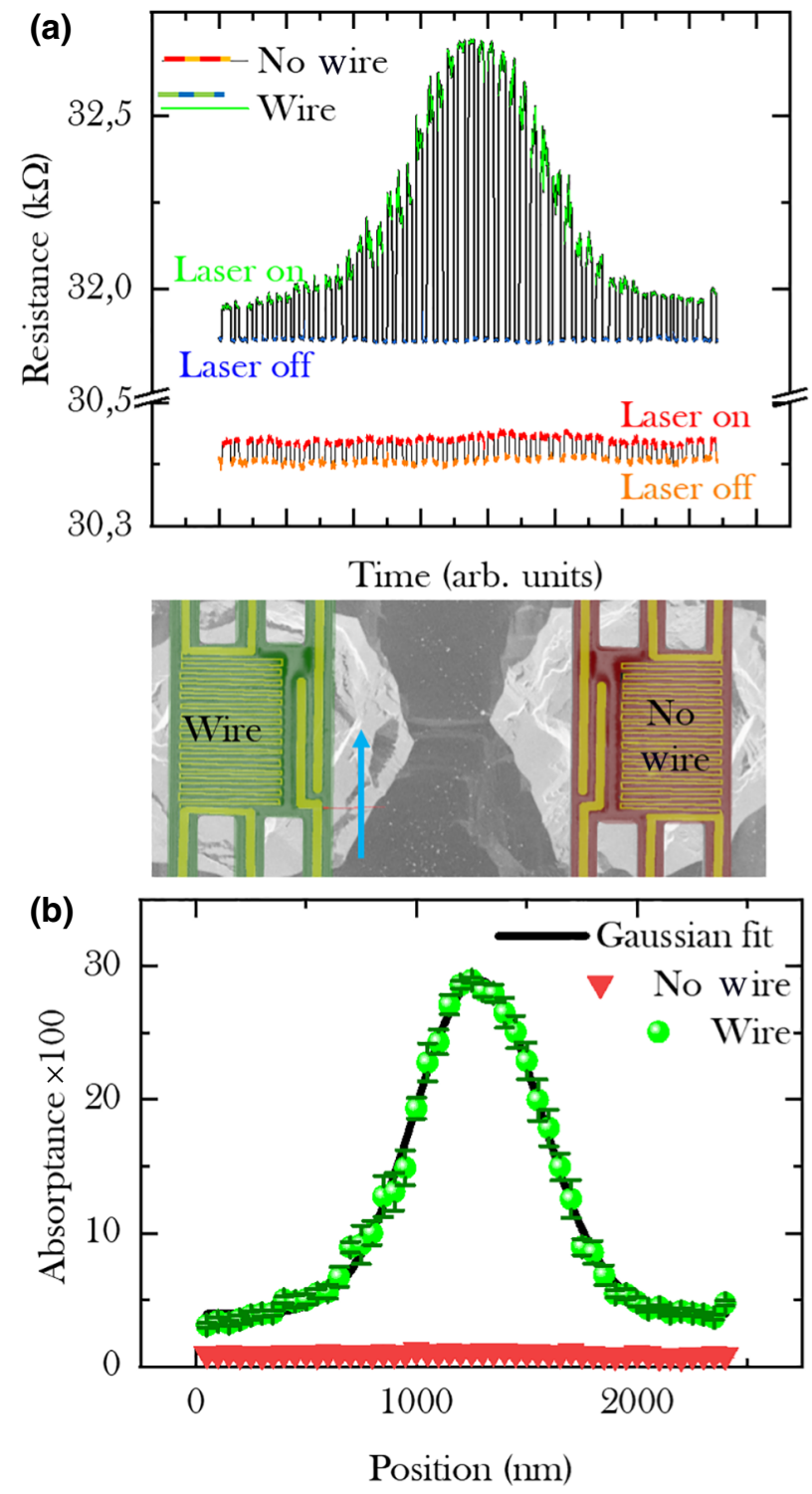

FIG. 2. Typical measurement. (a) Resistance of the thermometers as a function of time while the laser is being periodically turned on and off, and is moved perpendicularly to the NW. The "wire" and "no wire" resistances correspond to the resistance measured simultaneously on the platform with the suspended NW and the platform across, without any NW (see inset). (b) Absorptance calculated from the change in resistance between laser on and laser off configurations as a function of position. In green (red) is the absorptance as calculated for the platform with (without) any NW. The absorptance of the "no wire" configuration is coming from the scattered light from the substrate $P_{\text {back }}$. The background for the green points is higher because the laser spot is closer to this membrane, so scattering to this membrane is more intense. The black line is a Gaussian fit of this absorptance.

Apart from power directly absorbed by the NW, there is also laser light that is reflected from the underlying silicon substrate and consequently absorbed by the membranes.
This background power is measured with the laser spot far from the NW, but at the same distance from the membrane. To verify that the background power does not depend on the position of the laser beam with respect to the NW, the absorbed power is measured simultaneously on the opposite membrane. Since the NW is not connected to this membrane, only background radiation contributes to its heating. An absorbed background power independent of laser-spot position is found in this measurement, showing negligible influence from radiation from the wire, or shadowing of the reflection from the substrate by the wire. Therefore, a constant background is used for analyzing the absorption measurements.

\section{EXPERIMENTAL RESULTS}

\section{A. GaAs crystal structure dependence}

The absorptance of the $\mathrm{ZB}$ GaAs NW is shown in Fig. 4(a) for both the parallel and the perpendicular polarization. From this data, we notice an almost factor 5 lower absorptance for the perpendicular polarization compared to parallel. This can be attributed to the dielectric mismatch with the environment, which strongly suppresses absorption in the perpendicular direction [48]. However, despite the quite different absolute values of the parallel and perpendicular absorption, they exhibit nearly the same dependence of energy: the absorption is almost constant from 2 to $2.2 \mathrm{eV}$, then increases from 2.2 to $2.4 \mathrm{eV}$, and has a further, small increase at $2.6 \mathrm{eV}$. This common trend of the two polarizations suggests the absence of optical resonances. The only feature observable, the increase between 2.2 and $2.4 \mathrm{eV}$, can be attributed to an indirect transition from the second valence band to the $\mathrm{X}$ point in the conduction band [49].

When compared with the FDTD simulations [see Figs. 3(a) and 3(b)], we see that both simulations and experiments show an increase of the absorption around 2.4 $\mathrm{eV}$ for both polarizations. However, the magnitude and relative polarization dependence strongly depends on other parameters, like, e.g., thickness of the native oxide surrounding the NW, and spot size (for more simulations see Appendix B), that are hard to accurately determine. To further elucidate the dependencies we perform simulations for different oxide thicknesses. It can be seen that especially the higher-energy absorption of the perpendicular polarized light strongly depends on this oxide thickness, since perpendicular excitations are the most sensitive to the dielectric mismatch. The experimental data can be well matched to the simulations by choosing the appropriate oxide thickness, apart from the low-energy side of the parallel polarization. Since the simulations show an increase of absorption with decreasing energy for the parallel polarization, this can only be attributed to an optical resonance, which depends strongly on experimental conditions that are hard to control and that cannot be completely taken into 

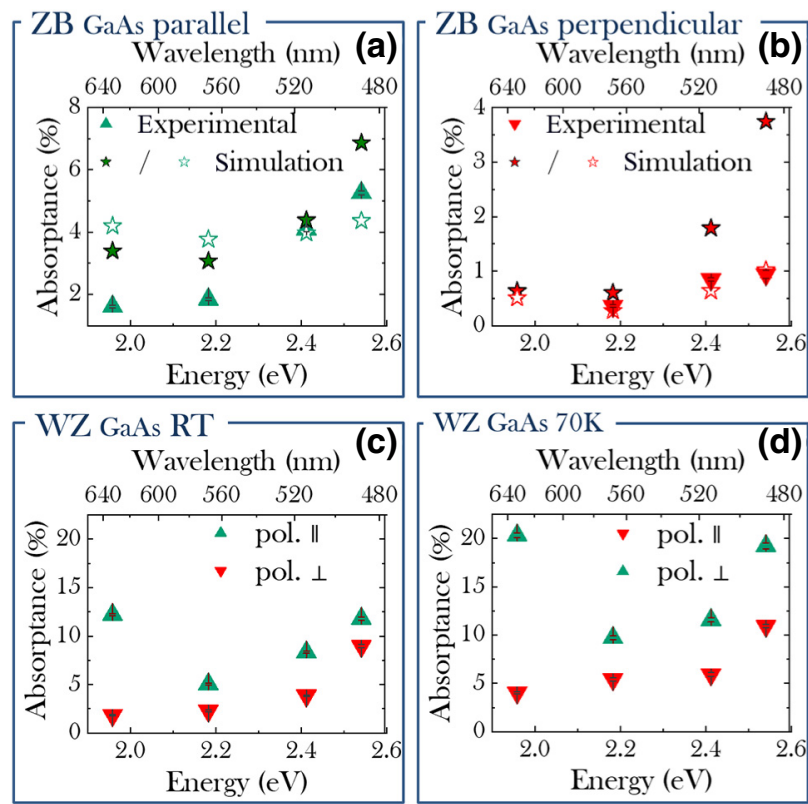

FIG. 3. Measured (triangles) and simulated (stars) absorptance of both ZB (a) and (b) and WZ GaAs (c) and (d). Green symbols correspond to experimental and simulated absorption with the incoming light parallel to the NW axis, while red symbols correspond to a perpendicular polarization. (a) Results for ZB GaAs NWs with the polarization axis parallel to the NW axis. The simulations are performed using a different thickness of the native oxide shell around the crystalline NW (full stars, no oxide shell; open stars, 5-nm thick shell) keeping the total diameter equal to that used in the experiments. (b) Same as (a) but now for the perpendicular polarization. (c) Experimentally determined absorptance for WZ GaAs at room temperature for both polarizations. (d) Results of measurements on the same wire as in (c) at $70 \mathrm{~K}$.

account in a simulation. The sensitivity of the simulations on parameters that are difficult to control or verify in practice further underpins the importance of measuring these parameters directly. The optical properties of ZB GaAs are relatively well known compared to all other materials studied in this work. This is due to the fact that the other materials have crystal structures that do not exist in bulk, resulting in much less known optical properties. Therefore, reliable FDTD simulations cannot be made for the investigated materials and are thus only performed for ZB GaAs, another motivation for quantitative experimental studies. Furthermore, even when experimental studies are performed, these are very sensitive to the exact experimental conditions such as spot size and scattering angle. Measuring the absorption in situ further ensures that the absorption is measured in the conditions relevant to the experiments.

When interpreting light absorption in NWs or nanorods, it is important to consider that a nonmonotonic behavior of the absorption can arise from electronic transitions of the material as well as from optical resonances, due to the fact that NWs can behave like an antenna. These resonances depend mainly on the NW size, the refractive index of the NW and of the medium surrounding it, and on the excitation wavelengths. A different thickness of the oxide shell surrounding the NW can, therefore, result in different resonance conditions. In general, peaks in the absorption that do not correspond to transitions to the fundamental or highenergy electronic band gaps can be reasonably attributed to optical resonances. If FDTD simulations can be performed, the dependence of their results on the parameters mentioned above is a clear indication of the presence of optical resonances. We carry out this type of study in Figs. 3(a) and 3(b) and Appendix C.

We now focus our attention on the comparison of the experimental results for the two different crystal structures of GaAs, as shown in Fig. 3. When we compare the results obtained from the ZB NW with results from a WZ NW [shown in Fig. 4(b)], it can be seen that the overall absorptance for the parallel polarization is similar between the $\mathrm{WZ}$ and $\mathrm{ZB}$. However, the increase in absorption observed for $\mathrm{ZB} \mathrm{GaAs}$ between 2.2 and $2.4 \mathrm{eV}$ disappears. Indeed, changing the crystal symmetry also alters the band structure, and a $\Gamma_{7} \rightarrow \Gamma_{7}(\mathrm{C})$ transition at $1.982 \mathrm{eV}$ is observed for WZ GaAs [50], which explains the increase at 1.959 $\mathrm{eV}$ for the parallel polarization. Apart from this point at $1.959 \mathrm{eV}$ the absorptance now depends on polarization less than for ZB. This can be attributed to the fact that the anisotropy is generally less for the WZ crystal structure compared to $\mathrm{ZB}$, due to a competition between the dielectric effect (favoring parallel polarization) and the intrinsic band-structure-related anisotropy (favoring perpendicular polarization) [48].

Finally, the WZ NW is also measured at $70 \mathrm{~K}$. At this temperature the energy dependence of the absorption is similar to the one measured at room temperature, but at

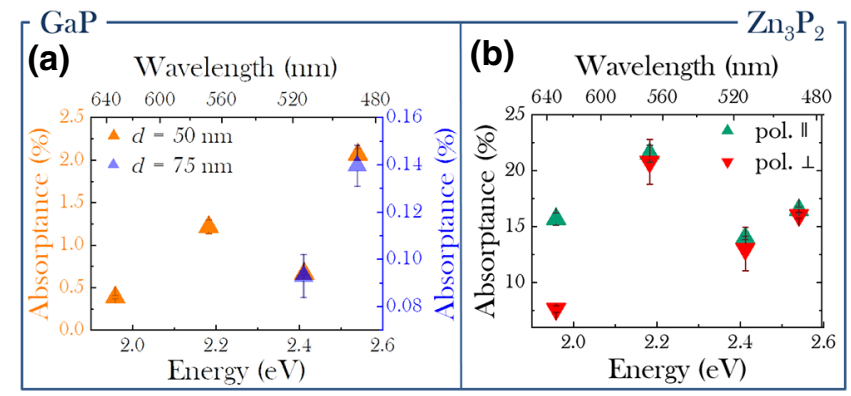

FIG. 4. Room temperature measured absorptance as a function of excitation energy, with excitation polarization parallel (upwards triangles) and perpendicular (downwards triangles) to the NW axis. (a) Results on two different WZ GaP NWs with a diameter of $50 \mathrm{~nm}$ (orange triangles, left axis) and $75 \mathrm{~nm}$ (blue triangles, right axis), both measured with light polarized parallel to the NW axis. (b) Results on the $\mathrm{Zn}_{3} \mathrm{P}_{2}$ superlattice NW. 
$70 \mathrm{~K}$ we observe an increase of its values. In principle, the energy of the electronic transitions of III-V semiconductors does not shift much from 0 to $70 \mathrm{~K}$ and therefore our result can be compared to the existing literature at 5-10 K [51,52]. However, there is no available literature on absorption (or PL and PLE) in wurtzite GaAs in the 2-2.6 eV range. We stress that the absorption of bulk semiconductors far from electronic transitions usually does not increase with decreasing temperature. Therefore, we attribute the observed increase either to the specific band structure of wurtzite GaAs or to effects related to the NW geometry, such as optical resonances or the presence of the native oxides surrounding the NW. The absence of literature available on the temperature-dependent absorption of WZ GaAs in the visible range and on the influence of the NW-specific geometry on the amount of absorbed light confirms that it is crucial to directly measure absorption in semiconductor NWs

\section{B. Wurtzite GaP}

Figure 4(a) shows the absorptance of WZ GaP NWs with a diameter of $50 \mathrm{~nm}$ (orange) and $75 \mathrm{~nm}$ (blue). Since the absorptance of this material is much lower than of the other materials, and the suppression in the perpendicular polarization is expected to be even stronger for these diameters, the absorption for the perpendicular polarization is below the sensitivity of this method. As can be seen from Fig. 4(c) the absorptance is much higher for the 50-nm case than for the $75 \mathrm{~nm}$, contrary to expectation. This is most likely caused by optical resonances for the 50-nm NW. Furthermore, for both diameters an increase of the absorptance between 2.4 and $2.6 \mathrm{eV}$ is observed, which can be reasonably expected to happen beyond the band gap. A band gap below $2.4 \mathrm{eV}$ at room temperature is in agreement with recent low-temperature PLE measurements [53,54]. The 50-nm NW also shows a small but noticeable increase at $2.2 \mathrm{eV}$, which is likely caused by an optical resonance, since this increase cannot be seen for the 75-nm NW, while it means a signal above noise level again. Finally, the $75 \mathrm{~nm}$ shows that even an absorptance below 0.001 can still be measured accurately using this method, corresponding to an absorbed power of approximately $12 \mathrm{nW}$. This clearly testifies the outstanding sensitivity that can be achieved using this method.

\section{C. $Z_{n_{3}} P_{2}$ superlattice}

The experimentally measured absorptance for the $\mathrm{Zn}_{3} \mathrm{P}_{2}$ superlattice NW is shown in Fig. 4(b). Despite having a very similar diameter to the NWs made by the less abundant semiconductor GaAs, this system shows the highest absorptance among the measured NWs, which suggests that its use in solar cells is very promising. Furthermore, the absorptance has only weak dependence on the energy within the measured energy range. This is expected since the measurements are taking place far beyond the band-gap energy $[35,36]$. The highest absorption, for both polarization configurations, is found at around $2.2 \mathrm{eV}$, which could be due to an electronic resonance, since theoretical studies predict a transition around this energy level [55]. However, we cannot exclude the contribution of an optical resonance. The absorptance is also independent of polarization, apart from a slight dependence at $1.95 \mathrm{eV}$. This could be due to the superlattice possibly causing light confinement, along the NW axis, and/or be a result of resonances polarized perpendicularly to the NW axis. To further explore this hypothesis it would be interesting to study the effect of the geometry separately, but this investigation goes beyond the scope of this work.

\section{SUMMARY AND COMPARISON WITH OTHER METHODS}

In this work a method to measure the optical absorption of single NWs is presented. The method is based on measurements of the temperature rise of a platform collecting the heat from a NW exposed to laser radiation. Here, the method is applied using a range of representative wavelengths from red to blue, and it can easily be extended to measure a full absorption spectrum. We also perform measurements at different temperatures, i.e., room temperature as well as $70 \mathrm{~K}$, to prove the applicability of the method in a wide temperature range. The minimum measurable power can be calculated from the minimum measurable temperature rise and the beam conduction using $P_{\min }=$ $G_{b} T_{\min }$. With a temperature sensitivity of about $100 \mathrm{mK}$, and a beam conduction of the order of $50 \mathrm{nW} / \mathrm{K}$, this leads to a minimum measurable absorbed power of about $5 \mathrm{nW}$, as also demonstrated by accurately measuring an absorbed power of $12 \mathrm{nW}$ in GaP NWs with a diameter of $75 \mathrm{~nm}$. This resolution is limited by the noise in the preamp, which is expected to be true even at very low temperatures [30]. Note that this sensitivity is also a factor- 2 improvement with respect to previous work [23] thanks to the simplified geometry where only one platform is used. Moreover, this configuration reduces fabrication errors that can lead to an asymmetry in the planarity of the platforms, inducing bending and possibly strain in the nanostructures.

Apart from the improved sensitivity with respect to some other methods, this method is also not limited in the temperature range. Measuring absorption at very low temperatures also requires negligible laser heating so that the sample temperature is very close to the bath temperature. Accurate measurements even with very low laser power are enabled by the high sensitivity discussed before. Therefore, using this method, the optical absorption can be measured for temperatures ranging from $\mathrm{mK}$ to the melting temperature of the system. This cannot be achieved using other methods, based on phase transitions [24], methods that cannot be applied inside a cryostat [25], or methods 
in which the laser power cannot be reduced sufficiently to avoid significant laser heating [29]. The wide temperature range means the method can be applied to systems that are interesting for light emission, sensing, as well as photovoltaics. Combined with the ability to measure sub-bandgap absorption more information on the optical properties of recently synthesized material systems can be obtained.

Beyond what we already achieve with the results presented in this work, the method can be further improved in terms of temperature range and measurement sensitivity, using solutions that have already been applied in other contexts. The resolution can be further improved by decreasing the beam conduction by making thinner and longer beams, or by improving the temperature sensitivity. A common way to improve the temperature sensitivity of these systems is by using a Wheatstone configuration [45], which has been shown to increase the temperature and consequently also the power sensitivity by 3 orders of magnitude, bringing it down to the $\mathrm{pW}$ range. This even enables the measurement of the absorption in single molecules with a very low-absorption cross section, such as Rh6G [28]. Furthermore, the temperature range can be extended by using an appropriate choice of metal. The method currently uses platinum resistive thermometers, which function most accurately at temperatures above $50 \mathrm{~K}$, the temperature range can be extended easily by using different materials for the thermometers such as $\mathrm{NbN}$ for temperatures down to $\mathrm{mK}[30]$.

\section{ACKNOWLEDGMENTS}

I.Z. has received funding from the European Research Council (ERC) under the European Union's Horizon

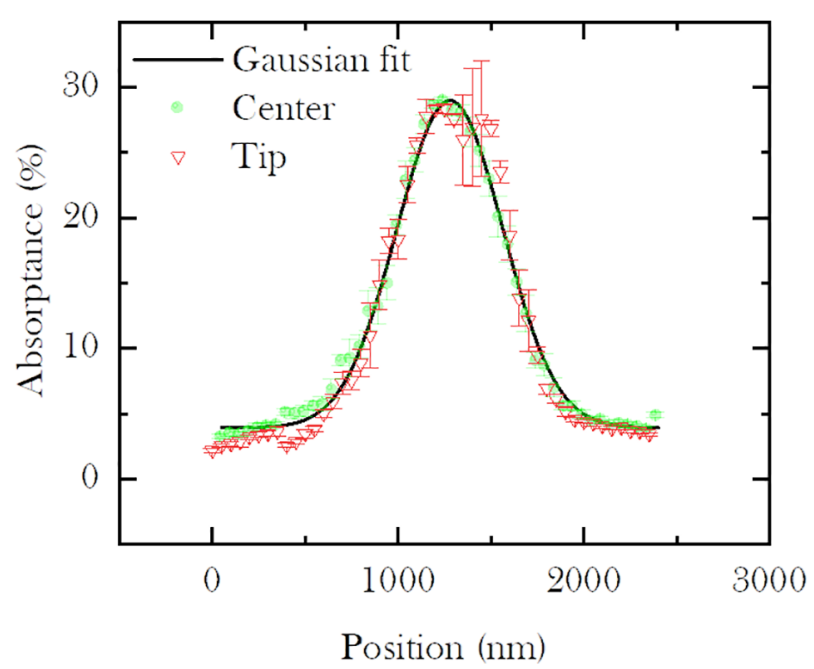

FIG. 5. Measured absorptance as a function of position with the laser spot moving either over the center of the wire (green circles) or over the tip of the wire (red triangles). The black line is a Gaussian fit of the data for the central position.
2020 research and innovation program (Grant Agreement No. 756365) and Swiss National Science Foundation research grant (Project Grant No. 200021 165784). A.F.M. acknowledges funding from the Swiss National Science Foundation research grant (Project Grant No. BSCGI0157705 ) and H2020 program through grant INDEED. H.D. acknowledges financial support by the Ministry of Education, Youth and Sports of the Czech Republic under the project CEITEC 2020 (LQ1601). M.D.L. acknowledges support from the Swiss National Science Foundation under the Ambizione grant (Grant No. PZ00P2 179801).

\section{APPENDIX A: POSITION DEPENDENCE}

To verify that the method does not depend on the conductance of the wire we measure the absorptance of a wire for two different positions; one near the center of the wire and one near the tip of the wire (ensuring that the entire laser spot is still on the wire). If the method depends on the thermal resistance between the laser and the membrane, moving the spot further from the membrane increases the thermal resistance and thus should show a change in absorptance. Instead, from Fig. 5 it can be seen that the two measurements give exactly the same result, demonstrating the validity of the model used.

\section{APPENDIX B: POWER DEPENDENCE}

To verify that the signal is truly coming from the laser and not from anything else, we also perform a laser-power dependence. We perform the experiment using the WZ GaAs NW, and the laser with a wavelength of $488 \mathrm{~nm}$, polarized parallel to the NW axis. We change the laser

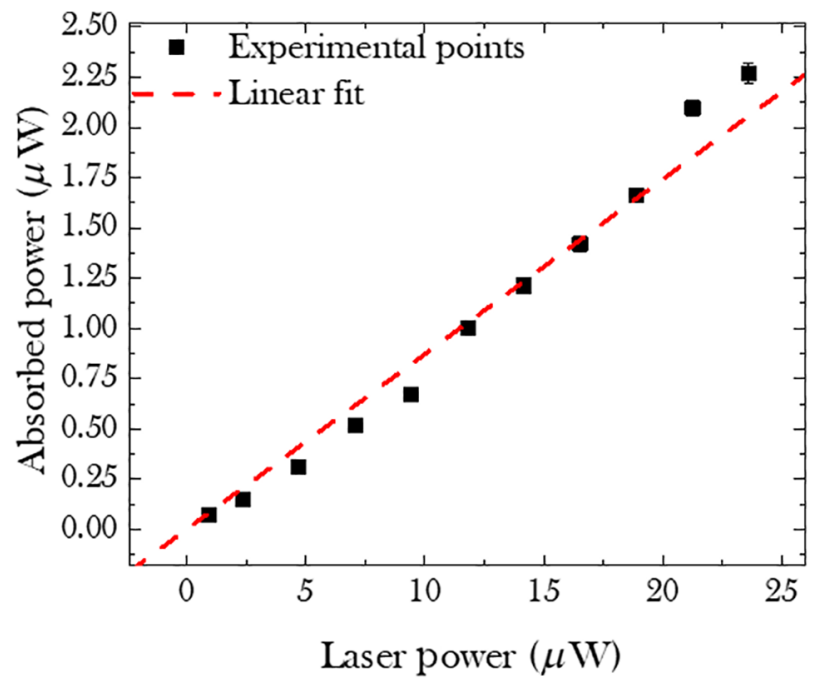

FIG. 6. Power-dependence measurement performed on a WZ GaAs NW, using a 488-nm laser polarized parallel to the NW axis, the black dots show the experimental results, while the red dashed line shows a linear fit with the intercept fixed at 0 . 


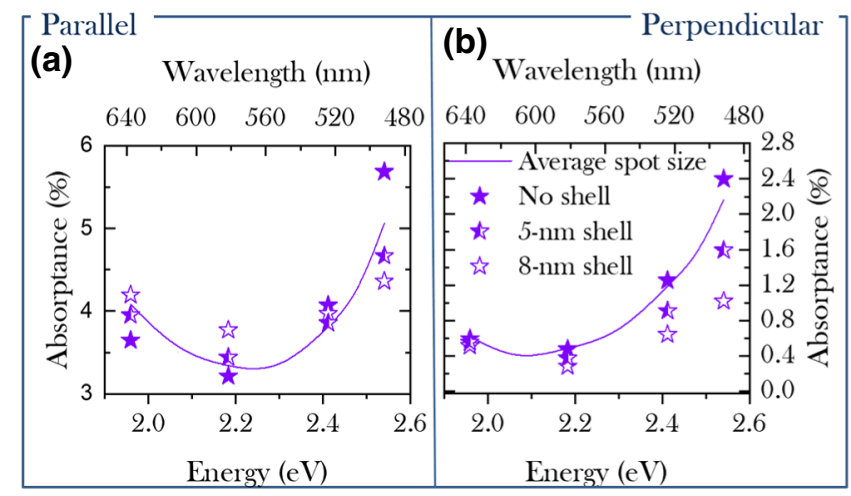

FIG. 7. Simulated absorptance as a function of energy for a ZB GaAs NW with a diameter of $70 \mathrm{~nm}$. The symbols are assuming the real, diffraction limited spot size, while the line is assuming an average among these spot sizes to show the true energy dependence. The full symbols represent simulations without any oxide shell, the half filled symbols a 5-nm oxide shell, and the empty symbols an 8-nm oxide shell. The line for the average spot size is simulated without any oxide shell.

power from 2 to $50 \mu \mathrm{W}$, resulting in a change in incoming laser power of approximately 1 to $25 \mu \mathrm{W}$. The result of this experiment is shown in Fig. 6. As seen from this plot, the absorbed varies linearly as a function of incoming power, with an absorption of approximately 0.1 . This value is slightly lower than the value reported in the wavelength dependence, which could be caused by minor alignment differences.

\section{APPENDIX C: SIMULATIONS}

In order to further investigate the dependence of the absorptance on experimental parameters we also perform simulations on a ZB GaAs NW for an average laser spot size, instead of the real wavelength-dependent laser spot size; and for another oxide thickness. The results of this study are shown in Fig. 7. As seen, the influence of the laser spot size for these conditions are relatively small, which justifies plotting our results as an energy dependence. The thicker oxide shell further enforces the trends already discussed in the main paper: the oxide shell plays the largest role when there are optical resonances, and an increasing oxide thickness can lead to either an increase or decrease of the absorption, depending on the energy.

[1] M. S. Gudiksen, L. J. Lauhon, J. Wang, D. C. Smith, and C. M. Lieber, Growth of nanowire superlattice structures for nanoscale photonics and electronics, Nature 415, 617 (2002).

[2] O. Gül, D. J. Van Woerkom, I. Van Weperen, D. Car, S. R. Plissard, E. P. Bakkers, and L. P. Kouwenhoven, Towards high mobility InSb nanowire devices, Nanotechnology 26, 0 (2015).
[3] S. Benter, V. G. Dubrovskii, M. Bartmann, A. Campo, I. Zardo, M. Sistani, M. Stöger-Pollach, S. Lancaster, H. Detz, and A. Lugstein, Quasi one-dimensional metalsemiconductor heterostructures, Nano Lett. 19, 3892 (2019).

[4] N. I. Goktas, P. Wilson, A. Ghukasyan, D. Wagner, S. McNamee, and R. R. LaPierre, Nanowires for energy: A review, Appl. Phys. Rev. 5, 0 (2018).

[5] M. Y. Swinkels and I. Zardo, Nanowires for heat conversion, J. Phys. D: Appl. Phys. 51, 0 (2018).

[6] E. Lai, W. Kim, and P. Yang, Vertical nanowire array-based light emitting diodes, Nano Res. 1, 123 (2008).

[7] Y. Cui, J. Wang, S. R. Plissard, A. Cavalli, T. T. Vu, R. P. Van Veldhoven, L. Gao, M. Trainor, M. A. Verheijen, J. E. Haverkort, and E. P. Bakkers, Efficiency enhancement of InP nanowire solar cells by surface cleaning, Nano Lett. 13, 4113 (2013).

[8] M. De Luca, Addressing the electronic properties of III-V nanowires by photoluminescence excitation spectroscopy, J. Phys. D: Appl. Phys. 50, 0 (2017).

[9] A. Standing, S. Assali, L. Gao, M. A. Verheijen, D. Van Dam, Y. Cui, P. H. Notten, J. E. Haverkort, and E. P. Bakkers, Efficient water reduction with gallium phosphide nanowires, Nat. Commun. 6, 1 (2015).

[10] N. Rossi, F. R. Braakman, D. Cadeddu, D. Vasyukov, G. Tütüncüoglu, I. M. A. Fontcuberta, and M. Poggio, Vectorial scanning force microscopy using a nanowire sensor, Nat. Nanotechnol. 12, 150 (2017).

[11] L. Cao, J. S. White, J. S. Park, J. A. Schuller, B. M. Clemens, and M. L. Brongersma, Engineering light absorption in semiconductor nanowire devices, Nat. Mater. 8, 643 (2009).

[12] S. L. Diedenhofen, O. T. Janssen, G. Grzela, E. P. Bakkers, and J. Gómez Rivas, Strong geometrical dependence of the absorption of light in arrays of semiconductor nanowires, ACS Nano 5, 2316 (2011).

[13] D. Spirkoska, J. Arbiol, A. Gustafsson, S. Conesa-Boj, F. Glas, I. Zardo, M. Heigoldt, M. H. Gass, A. L. Bleloch, S. Estrade, M. Kaniber, J. Rossler, F. Peiro, J. R. Morante, G. Abstreiter, L. Samuelson, and A. Fontcuberta i Morral, Structural and optical properties of high quality zinc-blende/wurtzite GaAs nanowire heterostructures, Phys. Rev. B - Condens. Matter Mater. Phys. 80, 1 (2009).

[14] K. A. Dick, C. Thelander, L. Samuelson, and P. Caroff, Crystal phase engineering in single InAs nanowires, Nano Lett. 10, 3494 (2010).

[15] L. Vincent, G. Patriarche, G. Hallais, C. Renard, C. Gardès, D. Troadec, and D. Bouchier, Novel heterostructured Ge nanowires based on polytype transformation, Nano Lett. 14, 4828 (2014).

[16] S. Assali, I. Zardo, S. Plissard, D. Kriegner, M. A. Verheijen, G. Bauer, A. Meijerink, A. Belabbes, F. Bechstedt, J. E. Haverkort, and E. P. Bakkers, Direct band gap wurtzite gallium phosphide nanowires, Nano Lett. 13, 1559 (2013).

[17] J. Panda, A. Roy, M. Gemmi, E. Husanu, and A. Phys, Electronic band structure of wurtzite $\mathrm{GaP}$ nanowires via resonance raman spectroscopy, Appl. Phys. Lett. 023108, 1 (2013). 
[18] H. I. T. Hauge, M. A. Verheijen, S. Conesa-Boj, T. Etzelstorfer, M. Watzinger, D. Kriegner, I. Zardo, C. Fasolato, F. Capitani, P. Postorino, S. Kölling, A. Li, S. Assali, J. Stangl, and E. P. Bakkers, Hexagonal silicon realized, Nano Lett. 15, 5855 (2015).

[19] C. Fasolato, M. De Luca, D. Djomani, L. Vincent, C. Renard, G. Di Iorio, V. Paillard, M. Amato, R. Rurali, and I. Zardo, Crystalline, phononic, and electronic properties of heterostructured polytypic Ge nanowires by Raman spectroscopy, Nano Lett. 18, 7075 (2018).

[20] P. Krogstrup, H. I. Jørgensen, M. Heiss, O. Demichel, J. V. Holm, M. Aagesen, J. Nygard, and A. Fontcuberta i Morral, Single-nanowire solar cells beyond the Shockley-Queisser limit, Nat. Photonics 7, 306 (2013).

[21] L. Gao, Y. Cui, J. Wang, A. Cavalli, A. Standing, T. T. Vu, M. A. Verheijen, J. E. Haverkort, E. P. Bakkers, and P. H. Notten, Photoelectrochemical hydrogen production on InP nanowire arrays with molybdenum sulfide electrocatalysts, Nano LettersNano Lett. 14, 3715 (2014).

[22] S. Yazji, M. Y. Swinkels, M. De Luca, E. A. Hoffmann, D. Ercolani, S. Roddaro, G. Abstreiter, L. Sorba, E. P. Bakkers, and I. Zardo, Assessing the thermoelectric properties of single InSb nanowires: The role of thermal contact resistance, Semicond. Sci. Technol. 31, 064001 (2016).

[23] I. K. Hsu, M. T. Pettes, A. Bushmaker, M. Aykol, L. Shi, and S. B. Cronin, Optical absorption and thermal transport of individual suspended carbon nanotube bundles, Nano Lett. 9, 590 (2009).

[24] C. Cheng, D. Fu, K. Liu, H. Guo, S. Xu, S. G. Ryu, O. Ho, J. Zhou, W. Fan, W. Bao, M. Salmeron, N. Wang, C. P. Grigoropoulos, and $\mathrm{J}$. Wu, Directly metering light absorption and heat transfer in single nanowires using metal-insulator transition in $\mathrm{VO}_{2}$, Adv. Opt. Mater. 3, 336 (2015).

[25] S. A. Mann, B. Sciacca, Y. Zhang, J. Wang, E. Kontoleta, H. Liu, and E. C. Garnett, Integrating sphere microscopy for direct absorption measurements of single nanostructures, ACS Nano 11, 1412 (2017).

[26] J. Wang, R. Shi, W. Wang, N. Cai, P. Chen, D. Kong, A. Amini, and C. Cheng, Directly probing light absorption enhancement of single hierarchical structures with engineered surface roughness, Sci. Rep. 8, 12283 (2018).

[27] M. Negri, L. Francaviglia, D. Dumcenco, M. Bosi, D. Kaplan, V. Swaminathan, G. Salviati, A. Kis, F. Fabbri, and A. Fontcuberta i Morral, Quantitative nanoscale absorption mapping: A novel technique to probe optical absorption of two-dimensional materials, Nano Lett. 20, 567 (2020).

[28] A. Gaiduk, M. Yorulmaz, P. V. Ruijgrok, and M. Orrit, Room-temperature detection of a single molecule's absorption by photothermal contrast, Science 330, 353 (2010).

[29] A. Crut, P. Maioli, N. Del Fatti, and F. Vallée, Optical absorption and scattering spectroscopies of single nanoobjects, Chem. Soc. Rev. 43, 3921 (2014).

[30] T. Nguyen, A. Tavakoli, S. Triqueneaux, R. Swami, A. Ruhtinas, J. Gradel, P. Garcia-Campos, K. Hasselbach, A. Frydman, B. Piot, M. Gibert, E. Collin, and O. Bourgeois, Niobium nitride thin films for very low temperature resistive thermometry, J. Low Temp. Phys. 197, 348 (2019).
[31] A. F. Oskooi, D. Roundy, M. Ibanescu, P. Bermel, J. D. Joannopoulos, and S. G. Johnson, Meep: A flexible free-software package for electromagnetic simulations by the FDTD method, Comput. Phys. Commun. 181, 687 (2010).

[32] K. Tomioka, J. Motohisa, S. Hara, K. Hiruma, and T. Fukui, GaAs/AlGaAs core multishell nanowire-based lightemitting diodes on Si, Nano Lett. 10, 1639 (2010).

[33] J. Greil, S. Assali, Y. Isono, A. Belabbes, F. Bechstedt, F. O. Valega MacKenzie, A. Y. Silov, E. P. Bakkers, and J. E. Haverkort, Optical properties of strained wurtzite gallium phosphide nanowires, Nano Lett. 16, 3703 (2016).

[34] S. Assali, J. Greil, I. Zardo, A. Belabbes, M. W. De Moor, S. Koelling, P. M. Koenraad, F. Bechstedt, E. P. Bakkers, and J. E. Haverkort, Optical study of the band structure of wurtzite GaP nanowires, J. Appl. Phys. 120, 0 (2016).

[35] S. Escobar Steinvall, N. Tappy, M. Ghasemi, R. R. Zamani, T. LaGrange, E. Z. Stutz, J.-B. Leran, M. Zamani, R. Paul, and A. Fontcuberta i Morral, Multiple morphologies and functionality of nanowires made from earth-abundant zinc phosphide, Nanoscale Horiz. 5, 274 (2020).

[36] G. M. Kimball, A. M. Müller, N. S. Lewis, and H. A. Atwater, Photoluminescence-based measurements of the energy gap and diffusion length of $\mathrm{Zn}_{3} \mathrm{P}_{2}$, Appl. Phys. Lett. 95, 3 (2009).

[37] Y. Calahorra, A. Husmann, A. Bourdelain, W. Kim, J. Vukajlovic-Plestina, C. Boughey, Q. Jing, A. Fontcuberta i Morral, and S. Kar-Narayan, Highly sensitive piezotronic pressure sensors based on undoped GaAs nanowire ensembles, J. Phys. D: Appl. Phys. 52, 0 (2019).

[38] L. Shi, D. Li, C. Yu, W. Jang, D. Kim, Z. Yao, P. Kim, and A. Majumdar, Measuring thermal and thermoelectric properties of one-dimensional nanostructures using a microfabricated device, J. Heat Transfer 125, 881 (2003).

[39] D. Li, Y. Wu, P. Kim, L. Shi, P. Yang, and A. Majumdar, Thermal conductivity of individual silicon nanowires, Appl. Phys. Lett. 83, 2934 (2003).

[40] R. Chen, A. I. Hochbaum, P. Murphy, J. Moore, P. Yang, and A. Majumdar, Thermal Conductance of Thin Silicon Nanowires, Phys. Rev. Lett. 101, 105501 (2008).

[41] A. Weathers and L. Shi, Thermal transport measurement techniques for nanowires and nanotubes, Annu. Rev. Heat Transfer 16, 101 (2013).

[42] M. Y. Swinkels, M. R. Van Delft, D. S. Oliveira, A. Cavalli, I. Zardo, R. W. Van Der Heijden, and E. P. Bakkers, Diameter dependence of the thermal conductivity of InAs nanowires, Nanotechnology 26, 385401 (2015).

[43] A. L. Moore and L. Shi, On errors in thermal conductivity measurements of suspended and supported nanowires using micro-thermometer devices from low to high temperatures, Meas. Sci. Technol. 22, 015103 (2011).

[44] J. Liu, H. Liu, Y. Hu, and X. Zhang, Differential laser flash Raman spectroscopy method for non-contact characterization of thermal transport properties of individual nanowires, Int. J. Heat Mass. Transfer 135, 511 (2019).

[45] M. C. Wingert, Z. C. Chen, S. Kwon, J. Xiang, and R. Chen, Ultra-sensitive thermal conductance measurement of onedimensional nanostructures enhanced by differential bridge, Rev. Sci. Instrum. 83, 024901 (2012). 
[46] A. V. Krasheninnikov and K. Nordlund, Ion and electron irradiation-induced effects in nanostructured materials, J. Appl. Phys. 107, 0 (2010).

[47] Q. Y. Li, J. H. Liu, H. D. Wang, X. Zhang, and K. Takahashi, Optical absorptance measurement of an individual multiwall carbon nanotube using a $\mathrm{T}$ type thermal probe method, Rev. Sci. Instrum. 84, 0 (2013).

[48] C. Wilhelm, A. Larrue, X. Dai, D. Migas, and C. Soci, Anisotropic photonic properties of III-V nanowires in the zinc-blende and wurtzite phase, Nanoscale 4, 1446 (2012).

[49] H. Ehrenreich, Band structure and electron transport of GaAs, Phys. Rev. 120, 1951 (1960).

[50] B. Ketterer, M. Heiss, E. Uccelli, J. Arbiol, and A. Fontcuberta i Morral, Untangling the electronic band structure of wurtzite GaAs nanowires by resonant Raman spectroscopy, ACS Nano 5, 7585 (2011).

[51] M. De Luca, G. Lavenuta, A. Polimeni, S. Rubini, V. Grillo, F. Mura, A. Miriametro, M. Capizzi, and F. Martelli,
Excitonic recombination and absorption in $\operatorname{In}_{x} \mathrm{Ga}_{1-x} \mathrm{As} /$ GaAs heterostructure nanowires, Phys. Rev. B - Condens. Matter Mater. Phys. 87, 1 (2013).

[52] L. Ahtapodov, J. Todorovic, P. Olk, T. Mjåland, P. Slåttnes, D. L. Dheeraj, A. T. Van Helvoort, B. O. Fimland, and $\mathrm{H}$. Weman, A story told by a single nanowire: Optical properties of wurtzite GaAs, Nano Lett. 12, 6090 (2012).

[53] S. Assali, Pure crystal phase nanowires: Growth and optical properties, September 2015, p. 190.

[54] B. C. da Silva, O. D. D. Couto, H. T. Obata, M. M. de Lima, F. D. Bonani, C. E. de Oliveira, G. M. Sipahi, F. Iikawa, and M. A. Cotta, Optical absorption exhibits pseudo-direct band gap of wurtzite gallium phosphide, Sci. Rep. 10, 7904 (2020).

[55] J. Andrzejewski and J. Misiewicz, Energy band structure of $\mathrm{Zn}_{3} \mathrm{P}_{2}$-type semiconductors: Analysis of the crystal structure simplifications and energy band calculations, Phys. Status Solidi (B) 227, 515 (2001). 